\title{
The pitfalls of permissive parenting
}

Volume 7 Issue 6 - 2017

\author{
Steven Richfield \\ Clinical Psychologist, Philadelphia, USA
}

\section{Opinion}

A parent writes, "One of our family's big challenges is the ongoing debate between my husband and I over how strict vs. how lenient we should be. Our kids complain that we are too strict, my husband complains that I am too flexible, and I complain that he is too rigid. This creates too much stress. How can we find a middle ground?"

Of all the necessary ingredients that parents add to the mixture called childrearing, rules and limits are among the most vital. Complicating this task, though, is the fact that excessive limits leads to the boiling over of resentment and defiance, but inadequate limits interfere with adaptation to rules and the willpower needed to resist unhealthy pressures. It's not uncommon for mothers and fathers to be on opposite sides of the "firmness fence," each convinced that the other is doing it wrong. This leads to inconsistencies, mixed messages about rules, and the undermining of each other's authority. Such circumstances can breed dishonesty, deceit and manipulation within children, some of the very behaviors that proper limits are designed to discourage and prevent. Therefore, it is particularly important that parents are united in their approach to this issue.

The authoritarian parenting style and permissive parenting style are on opposite ends of the spectrum. Here are some suggestions for finding the elusive middle ground

Bear in mind that upbringing plays a pivotal role in this clash of philosophies. The limits and punishments handed down by our parents create a template for what we refer to as parenting. Some of us defend our parenting decisions with the statement, "I turned out okay," as if this indicates that our kids will be just as happy and well adjusted. To borrow a phrase from the investing world, past results do not guarantee future performance. Today's complex culture has led to an entirely different array of forces and frustrations that parents must help equip their kids to contend with. Simply doing what was done to us risks overlooking many opportunities to use limits, coaching, and consequences to build stronger character strengths in our kids. One way to act upon this knowledge is to consider which past parenting lessons are helpful in today's world and which ones need discarding. Take heed of your spouse's opinions since to ignore those leads to troubling results for your children. Children who are raised with two different sets of limits and consequences have more difficulty adapting to the outside world. Rather than internalizing rules that become selfgoverning, they seek out fulfillment of their desires by deception, avoidance, and self -justification. This underscores what's at stake if parents don't resolve their differences. If you can't totally agree with your spouse's position consider what you "can live with" as the next best choice. The benefits of unified rules and consequences, even if you are somewhat unhappy with them, is preferred to the arbitrariness of shifting standards and attempts to "make up" for the perceived excesses of one's spouse. Remember that parenting often leads us directly to our triggers or hot spots. This is due to the expectations and emotions that we wrap tightly around our children's behavior. When they act out inappropriately, we are at risk for losing control over our reacting sides. This can be a major issue when couples don't agree about rules and discipline. One parent is responding emotionally to the child's misbehavior; the other parent attempts to shield the child from this fall out. The over -emotional parent is wise to consider where their triggers are in order to prepare a more thoughtful response. The other parent would be wise to use verbal diplomacy when discussing this loaded issue. Consider what mental blinders you might bring into your parenting role. These blinders get in the way of our seeing our child accurately or responding empathically. Sometimes it's due to behaviors in our child that remind us of parts of ourselves, siblings, or parents that we have associated with negative or hurtful memories. Sometimes the blinders are due to aspects in our spouse which we find undesirable and find evidence of in our child. If this is the case, it's likely contributing to an overly harsh or lenient disciplinary style. Try to have as open and honest a discussion with your spouse as you possibly can, recognize where these blinders may be emanating from, and pledge to find ways to shed them.

\section{Acknowledgments}

None.

\section{Conflicts of interest}

Author declares there are no conflicts of interest.

\section{Funding}

None. 